\title{
Blind structured illumination as excitation for super-resolution photothermal radiometry
}

\author{
by P. Burgholzer*, T. Berer*, M. Ziegler**, E. Thiel**, S. Ahmadi**, \\ J. Gruber ${ }^{\star \star \star}$, G. Mayr ${ }^{\star \star \star}$, G. Hendorfer ${ }^{\star \star \star}$ \\ * Research Center for Non Destructive Testing (RECENDT), Altenberger Straße 69, 4040 Linz, Austria, \\ E-mail: peter.burholzer@recendt.at \\ ** Bundesanstalt für Materialforschung und -prüfung (BAM), 12200 Berlin, Germany \\ *** Josef Ressel Centre for Thermal NDE of Composites, University of Applied Sciences Upper Austria, \\ Stelzhammerstr. 23, 4600 Wels, Austria
}

\begin{abstract}
Using an infrared camera for radiometric imaging allows the contactless temperature measurement of multiple surface pixels simultaneously. From the measured surface data, a sub-surface structure, embedded inside a sample or tissue, can be reconstructed and imaged when heated by an excitation light pulse. The main drawback in radiometric imaging is the degradation of the spatial resolution with increasing depth, which results in blurred images for deeper lying structures. We circumvent this degradation with blind structured illumination, combined with a non-linear joint sparsity reconstruction algorithm. The ground-breaking concept of super-resolution can be transferred from optics to thermographic imaging.
\end{abstract}

\section{Introduction}

In non-destructive imaging of opaque or turbid samples, the spatial information about subsurface structures (for example defects), can be extracted from measured surface data, such as acoustic pressure for ultrasonic imaging or temperature for thermographic imaging. The information about the defects is transferred from the samples interior to its surface by acoustic waves or heat diffusion, respectively. Recently, we showed that the entropy production of these propagation processes limits the transferred information [1, 2]. This poses a principle resolution limit, similar to the Abbe limit in optics. Thermodynamic fluctuations are reason for the entropy production, which reduces the available information about the subsurface structures in the measured surface data. They are extremely small for macroscopic samples, but are highly amplified due to the ill-posed problem of image reconstruction. For macroscopic samples, the resolution limit depends only on the amplitude of these fluctuations.

Diffusive processes show a high entropy production with increasing diffusion length. Therefore, the resolution limit for thermographic imaging is found to be proportional to the subsurface depth and is described by a depthdependent thermal point-spread-function (PSF) [2]. Similar to optics and acoustics, the blurring of imaged structures for thermographic imaging is modelled by convolution with this thermal PSF. Structures smaller than the width of the PSF cannot be resolved in conventional thermographic imaging. Circumventing such a principle resolution limit is called super-resolution. We discuss how blind (unknown) structured illumination combined with non-linear reconstruction algorithms using sparsity of the imaged structures allows thermographic imaging without high degradation of spatial resolution for deeper lying structures.

In optics, it is known since 2007 that optical diffusion in a strong scattering sample can be "inverted" by wavefront shaping [3]. Coherent photons from a laser scatter in a deterministic way (at least for a certain correlation time), which is measured point wise and the resulting scattering matrix is used to change the light wavefront e.g. by a spatial light modulator in such a way that after the diffusive scattering the light is focused. For heat diffusion, phonons are not coherent and the propagation of phonons is highly uncorrelated in time. Therefore, a "phonon scattering matrix" cannot be measured and used to invert heat diffusion in time.

By adequate structured illumination [4, 5, 6], the spatial frequencies of the imaged object are downshifted by frequency mixing, resulting from the multiplication of the illumination pattern with the spatially varying optical absorption coefficient of the object. These downshifted spatial frequencies can be imaged if they lie within the low-frequency passband given by the Fourier transform of the PSF. Usually, in structured illumination, reconstruction algorithms use information about the illumination patterns for calculating the images. However, even small errors in the patterns can lead to artefacts in the final images [5]. Therefore, blind structured illumination was proposed where the knowledge of the illumination pattern is not necessary. Here, it is assumed that illumination patterns are positive and their sum is uniform [5]. Recently, two reconstruction algorithms using joint support and sparsity (called also block sparsity) which were applied for acoustic-resolution photoacoustic microscopy [7, 8] and for thermographic imaging [9] were proposed.

In this work, it is shown that the degradation of the spatial resolution with increasing depth can be circumvented by using blind structured illumination, combined with a non-linear joint sparsity reconstruction algorithm. We demonstrate this by imaging a line pattern and a star-shaped structure through a metal sheet with a higher resolution than the width of 
the thermal PSF. The proof-of-principle of this approach was realized by a structured illumination using a flash lamp via parallel slits cut in an aluminum foil. Then, the structured illumination was realized by a VCSEL array which illuminates with pseudo-random line patterns. This direct type of illumination at a great distance can be seen as a step towards a measurement method that can actually be used productively. Both realizations of super-resolution thermographic imaging demonstrate that blind structured illumination allows thermographic imaging without high degradation of the spatial resolution for deeper lying structures. The ground-breaking concept of super-resolution can be transferred from optics to diffusive imaging by defining a thermal PSF, which gives the principle resolution limit for a certain signal-tonoise ratio, similar to the Abbe limit for a certain optical wavelength.

\section{Experimental set-up and iterative joint-sparsity reconstruction method}

What is imaged in thermographic imaging, when we illuminate a structure on a surface of a steel sheet, such as line pairs having a decreasing distance (Fig. 1, left)? On the rear sample side, the temperature evolution in time after the heating pulse is measured as a function of time. Various methods can be used to reconstruct the temperature on the front surface just after the heating pulse from the measured temperature evolution. We have used a virtual wave reconstruction method [10], where for a homogenous light flux $I$ the reconstructed thermographic image $y(r)$ is proportional to the convolution of the thermographic PSF $h(r)$ with absorbed light $\rho(r)$ (Fig. 1, right):

$$
y(r)=h(r) *[I(r) \cdot \rho(r)] .
$$

In Eq. (1) constant material factors such as the heat capacity are included in the thermographic PSF $h(r)$.

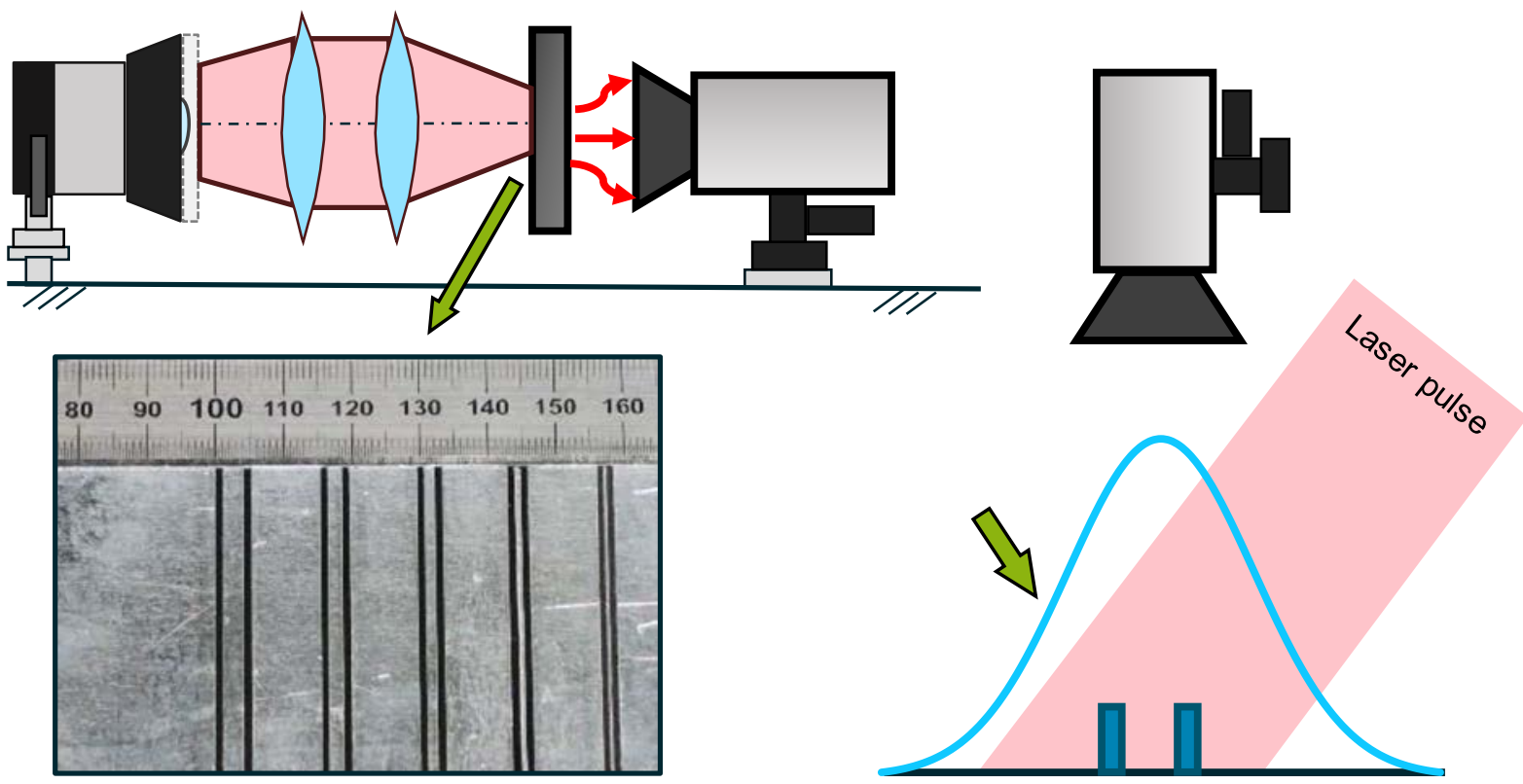

Fig. 1. Left. Homogenous illumination of a steel sheet by a short laser pulse, which has absorbing line patterns on its surface where the light heats the surface. Between the lines the light is reflected and nearly no heat is absorbed. The temperature evolution after the heating pulse is measured on the rear surface by an IR camera. Right: If the distance of a line pair is less than the width of the PSF $h(r)$ from the thermographic reconstruction the individual lines cannot be resolved.

To increase the spatial resolution, the light is focused on a small laser line, which scans the sample and does not illuminate both of the lines at the same time. Now, the spatial resolution is determined by the width of the optical line, which is smaller than the distance of the two lines (Fig. 2). In comparison to Fig. 1 the two lines can be resolved as two individual objects, if they are not both illuminated at the same time. The principle of super-resolution is that the localization of the maximum of the individual peak can be done with much higher precision than the width of the PSF, which is shown in Fig. 3. We could demonstrate this not only for known illumination patterns but also for unknown illumination, which is called blind structured illumination.

In optical microscopy, Gustafson achieved super-resolution by using spatially structured illumination in a widefield fluorescence microscope. The sample is illuminated with a series of excitation light patterns, which cause normally inaccessible high-resolution information to be encoded into the observed image. The recorded images are linearly 
processed to extract the new information and produce a reconstruction with up to twice the normal resolution. Even if the structured illumination pattern is known, the resolution enhancement in structured illumination microscopy is limited to about a factor of two because the maximum spatial frequency of the illumination pattern is constrained by the optical transfer function of the microscope [4].

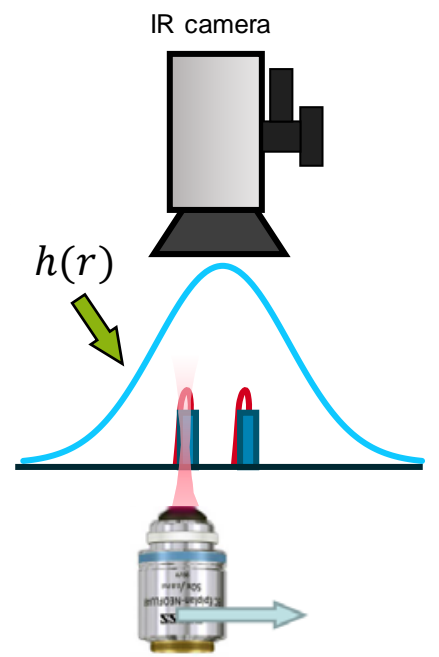

Fig. 2. Focused illumination of the laser pulse, which scans the sample surface. The spatial resolution is determined by the width of this line, which is smaller than the thermographic PSF $h(r)$. In comparison to Fig. 1 the two lines can now be resolved as two individual lines.

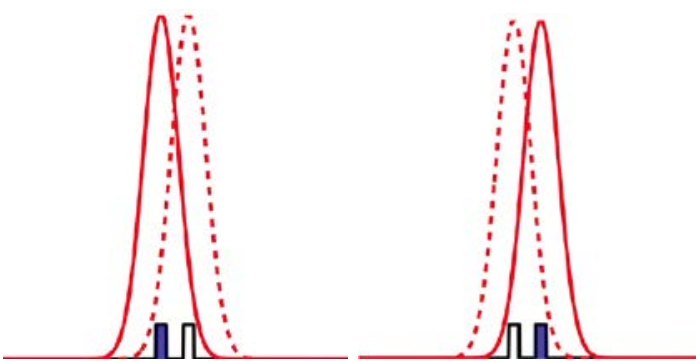

Fig. 3. Principle of super-resolution: the localization of the maximum of the individual peaks can be done with much higher precision than the width of the PSF

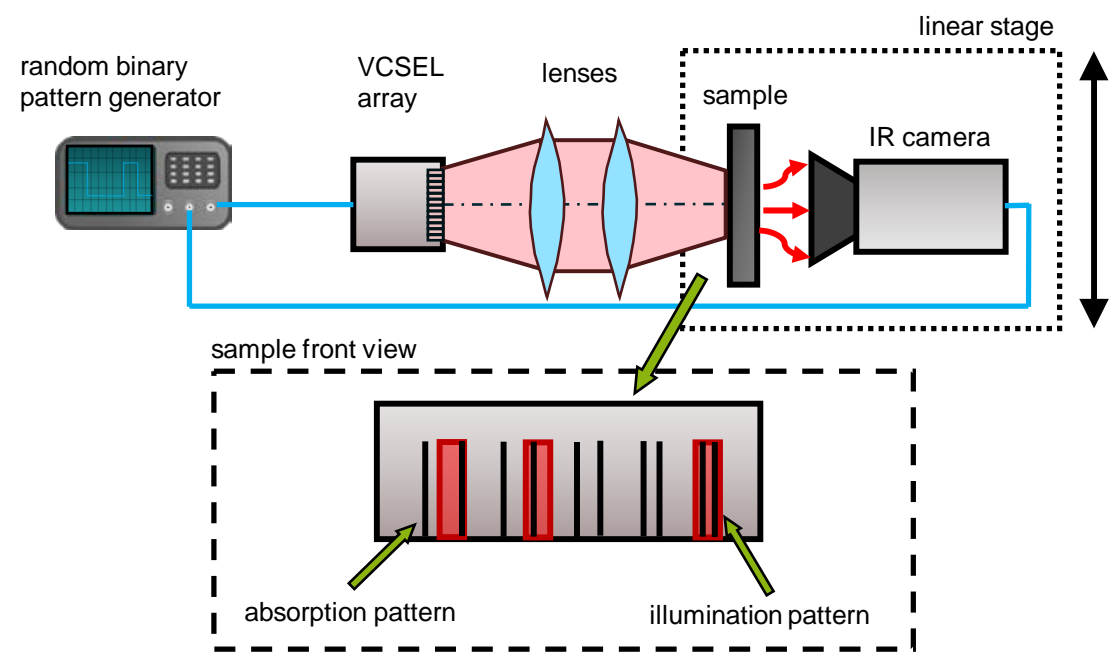

Fig. 4. Blind structured illumination generated by a random binary pattern generator controlling a VCSEL array. 
Our method for increasing the spatial resolution was inspired by recent work in fluorescence microscopy where super-resolution imaging was demonstrated using multiple unknown speckle illumination patterns [5, 6]. We extend this concept to thermographic imaging, with several illumination patterns, similar to what we have done for photoacoustic imaging [7]. As illustrated in Fig. 4, we use $M$ different illumination patterns $I_{1}, I_{2}, \ldots, I_{M}$ to illuminate the sample. The illumination patterns and the absorber distribution are represented by discrete vectors $I_{m}, \rho \in \mathbb{R}^{N}$, where the components denote values at equidistant points $x_{i}$ in the imaging domain. According to Eq. (1) the measured signal from the focused transducer is

$$
y_{m}=h *\left[I_{m} \cdot \rho\right]+\epsilon_{m} \text { for } m=1, \ldots, M .
$$

Here $\epsilon_{m}$ indicates the noise (error) in the data. The goal is to recover the absorber distribution $\rho$ and, to some extent, the illumination pattern $I_{m}$ from the data. The product $I_{m} \cdot \rho$ is the heating source just after the pulse corresponding to the $m$-th illumination pattern. The heating sources $T_{m} \triangleq I_{m} \cdot \rho$ are (theoretically) uniquely determined by the deconvolution equations $y_{m}=h * T_{m}+\epsilon_{m}$ for $m=1, \ldots, M$. However, due to the ill-conditioned nature of deconvolution with a smooth kernel, these uncoupled equations are sensitive to errors and further only provide lowresolution reconstructions when solved independently and without proper regularization. In order to obtain high-resolution reconstructions, we propose to use a joint-sparsity penalty, which uses the fact that all products $T_{m}=I_{m} \cdot \rho$ come from the same density distribution $\rho$ [7].

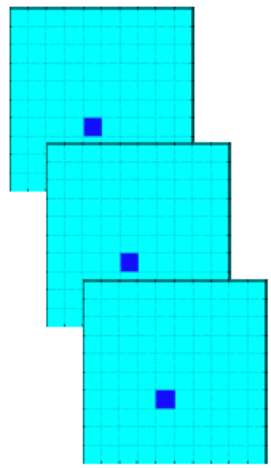

$\|T\|_{2,1}=10$

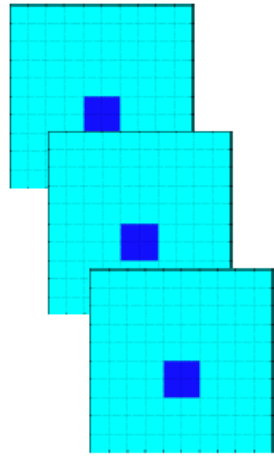

$\|T\|_{2,1}=40$

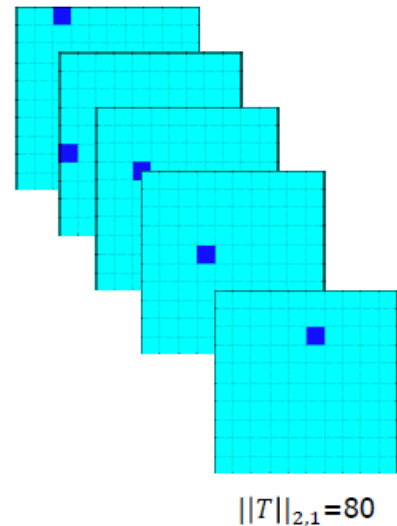

$\|T\|_{2,1}=80$

Fig. 5. Example to demonstrate how the joint-sparsity term in Eq. (4) prefers jointly sparse solutions. For simplification, only $N=10 \times 10=100$ pixel values and $M=100$ illumination patterns are used. $A$ dark pixel means a value of one; a light pixel is a value of zero. Left: Only one pixel per frame is set to one, and it is the same for every illumination pattern. For this dark pixel the $\ell^{2}$-norm over all $M$ different illumination patterns is 10 , for the others it is zero. Middle: Four such pixels with a constant value and a $\ell^{2}$-norm over all $M$ different illumination patterns of 10 exist, resulting in a total value of 40. Right: Similar to the left case only one pixel has a value of one, but now it is not the same pixel for every illumination pattern but randomly chosen. If all the pixel values would be pairwise different for every illumination pattern, the value of the joint-sparsity term $\|T\|_{2,1}$ would be 100 .

Numerically this was implemented by minimizing

$$
F(T)=\frac{1}{2} \sum_{m=1}^{M} \sum_{i=1}^{N}\left|h * T_{m}\left(x_{i}\right)-y_{m}\left(x_{i}\right)\right|^{2}+\alpha_{1}\|T\|_{2,1}+\frac{\alpha_{2}}{2}\|T\|_{2}^{2} \rightarrow \min _{T}
$$

using the FISTA (fast iterative thresholding algorithm) [11]. The first term in Eq. (3) is the data fitting term, the second term the joint-sparsity (or block-sparsity) inducing term and the last term is a stability term known from Tikhonov regularization. In the following, we show a small example of how the joint-sparsity term

$$
\|T\|_{2,1}=\sum_{i=1}^{N} \sqrt{\sum_{m=1}^{M}\left|T_{m}\left(x_{i}\right)\right|^{2}}
$$

yields solutions preferring joint sparsity. First, for every individual measured pixel the $\ell^{2}$-norm over all $M$ different illumination patterns is taken and then these positive values are summed up ( $N$ pixels). As this term is essential for our IJOSP algorithm its values for three different examples are shown in Fig. 5, for $N=10 \times 10=100, M=100$. It is shown by this simple example that this term favors joint (block) sparsity, meaning that it has a lower value for solutions which are sparse, but also if they have non-zero entries at the same pixel position for all the illumination patterns.

In the rest of this section more details about the two used experimental set-ups are given: A 3 mm thick steel sheet (common structural steel with a thermal diffusivity of $16 \mathrm{~mm}^{2} \mathrm{~s}^{-1}$ ) was painted black on both sides for light 
absorption and radiation purposes. On the front side, absorbing patterns such as parallel lines and a star-shaped pattern were created by using aluminum tape as a reflecting mask.

In our first experiment, the structured illumination was created by parallel slits cut in aluminum foil, where the excitation from a flashlight could penetrate. A slit mask with $1 \mathrm{~mm}$ wide slits at a distance of $10 \mathrm{~mm}$ to each other was moved in the horizontal $x$-direction with a step size of $0.2 \mathrm{~mm}$. The slits were not only oriented in the vertical $y$-direction, but also were also rotated $\pm 45^{\circ}$ in the $x$-y-plane. 55 patterns for each direction were used for illumination, resulting in 165 illumination patterns in total. As a result, only the unmasked (black) patterns behind the slits absorb light from the optical flash light excitation (xenon gas discharge tube PBC -6000 from Blaesing using $6 \mathrm{~kJ}$ electrical energy and 2 ms pulse duration). An infrared camera (Ircam Equus $81 \mathrm{k}$ M Pro) was used to measure the temperature evolution on the rear side.

In our second experiment the same sample was used but for generation of the structured illumination a VCSEL laser array (wavelength: $980+/-10 \mathrm{~nm}$, maximum output power: $2.4 \mathrm{~kW}$ ) with twelve individual emitter cells was used (see Fig. 4)[12,13]. The sample was placed together with the IR camera (Infratec ImageIR 8300, 640x512 pixel, spectral range $2-5 \mu \mathrm{m}, 100 \mathrm{~Hz}$ frame rate, spatial resolution: $132 \mu \mathrm{m} / \mathrm{pixel}$ ) on a linear stage. This linear stage allowed varying the position of the sample and the IR camera perpendicular to the optical axis with micrometer precision. The structural illumination was realized by a 1:1.125 optical projection of the VCSEL array via a lens pair (each $\mathrm{f}=250 \mathrm{~mm}$, distance between VCSEL and sample: $505 \mathrm{~mm}$ ) onto the sample surface leading to an irradiance of $70 \mathrm{~W} / \mathrm{cm}^{2}$. The twelve individual emitter cells were controlled by a pseudo-random binary pattern generator. The measurement system is automated such that the VCSEL array emits for each measurement a randomly created illumination pattern of $4.5 \mathrm{~mm}$ wide linear stripes with $0.28 \mathrm{~mm}$ distance. In this experiment ten different measurements based on randomly chosen illumination patterns are executed before a position shift of $0.225 \mathrm{~mm}$ was performed. Working with ten positions results in 100 measurements. It was ensured that at least one VCSEL array cell was switched on for each measurement. Moreover, the VCSEL array cells were active for $200 \mathrm{~ms}$ in each measurement (irradiance: $14 \mathrm{~J} / \mathrm{cm}^{2}$ ) in order to obtain a sufficiently high temperature rise on the sample. Between two measurements, the samples cooled down for $15 \mathrm{~s}$ (i.e. 1500 camera frames), resulting in an overall measurement duration of approximately 25 min. Although not performed thus far, it is easily possible to change the line thickness of the illumination pattern on the sample, by simply changing the optical magnification of the VCSEL array.

\section{Results and Discussion}

The theoretical lateral width (FHWM) of the thermographic PSF was evaluated to be $5.9 \mathrm{~mm}$ using the $3 \mathrm{~mm}$ thickness of the steel sheet and a signal-to-noise ratio (SNR) of 144 for the used thermographic measurements [7]. This could be experimentally validated. By using 55 different patterns of structured illumination and our iterative joint sparsity algorithm (IJOSP) [5], it was possible to resolve $1 \mathrm{~mm}$ thick lines at a distance down to $0.6 \mathrm{~mm}$, which results in a resolution enhancement of approximately a factor of four (Fig. 6). With "conventional" deconvolution (Richardson-LucyDeconvolution) the line pairs only down to a distance of $1.3 \mathrm{~mm}$ could be resolved, which demonstrates the capability of our super-resolution thermographic reconstruction method. The disadvantage is the higher number of structured illumination measurements, but for a fair comparison we have used for the "mean-signal" reconstruction and the Richardson-Lucy-Deconvolution the averaged signal of all 55 structured illumination pattern signals. The used measurement time is therefore equal for all compared methods. A similar enhancement in spatial resolution could be demonstrated by reconstructing the image of a two-dimensional star-shaped structure using 165 different patterns of structured illumination.

Using our second set-up, we are able to vary the illumination line width of our patterns by changing the magnification of the lenses in the illumination path (Fig. 4). In the present experiment, we chose an illumination line width of $4.5 \mathrm{~mm}$ such that all line pairs could be illuminated at once. Since now the illumination line width is much larger then with the flash lamp, the resolution of our iterative reconstruction turned out to be worse. It was possible to resolve the line pairs down to a distance of approximately $0.9 \mathrm{~mm}$, which corresponds to $20 \%$ of the illumination line width (Fig. 7 for an illumination line width of $4.5 \mathrm{~mm})$. The line pair with the smallest distance $(0.6 \mathrm{~mm}$ distance) could not be resolved and the second one $(0.9 \mathrm{~mm}$ distance) showed a strong asymmetry with one line being much stronger than the other. Although in principle such a remarkable resolution could be achieved, the signal to noise ratio is worse. However, when comparing the results of both experiments, we should note that in the VCSEL experiment a 200 ms pulse was used instead of the $2 \mathrm{~ms}$ long pulse in the flash experiment. Such a long pulse results in an additional broadening of the PSF which can be described by an additional convolution of the original PSF with this excitation pulse. As a result, the reconstructed PSF and the mean signal in Fig. 7 (solid blue line) is broadened by a factor of $\approx 2$. From this comparison it becomes clear that both experiments achieve a similarly good improvement of the resolution.

\section{Conclusions and Outlook}

In this work, it is shown that the loss in resolution for thermographic imaging proportional to the imaging depth can be circumvented by using blind structured illumination, combined with a non-linear joint sparsity reconstruction algorithm. We demonstrate this by imaging a line pattern and a star-shaped structure through a metal sheet with a resolution four times better than the width of the thermal point-spread-function. The structured illumination is realized by parallel slits cut in an aluminum foil, where the excitation is carried out either by a flashlight, which passes the foil through the slits, or by a VCSEL array illuminating pseudo-random line patterns. This realization of super-resolution 
thermographic imaging demonstrates that blind structured illumination allows thermographic imaging without high degradation of the spatial resolution for deeper lying structures. The ground-breaking concept of super-resolution can be transferred from optics to diffusive imaging by defining a thermal point-spread-function, which gives the principle resolution limit for a certain signal-to-noise ratio, similar to the Abbe limit for a certain optical wavelength.

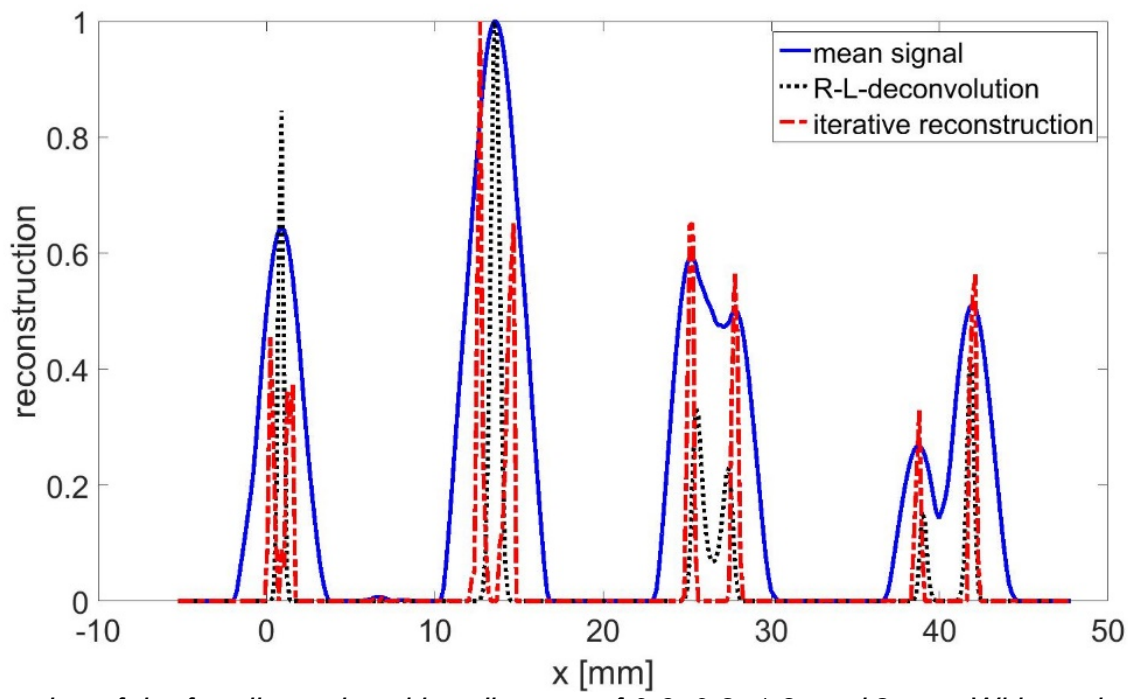

Fig. 6. Reconstruction of the four line pairs with a distance of $0.6,0.9,1.3$, and $2 \mathrm{~mm}$. With our iterative joint sparsity reconstruction all line pairs could be resolved, with the mean signal reconstruction and the Richardson-Lucy (R-L)-deconvolution the two nearest line pairs cannot be separated individually.

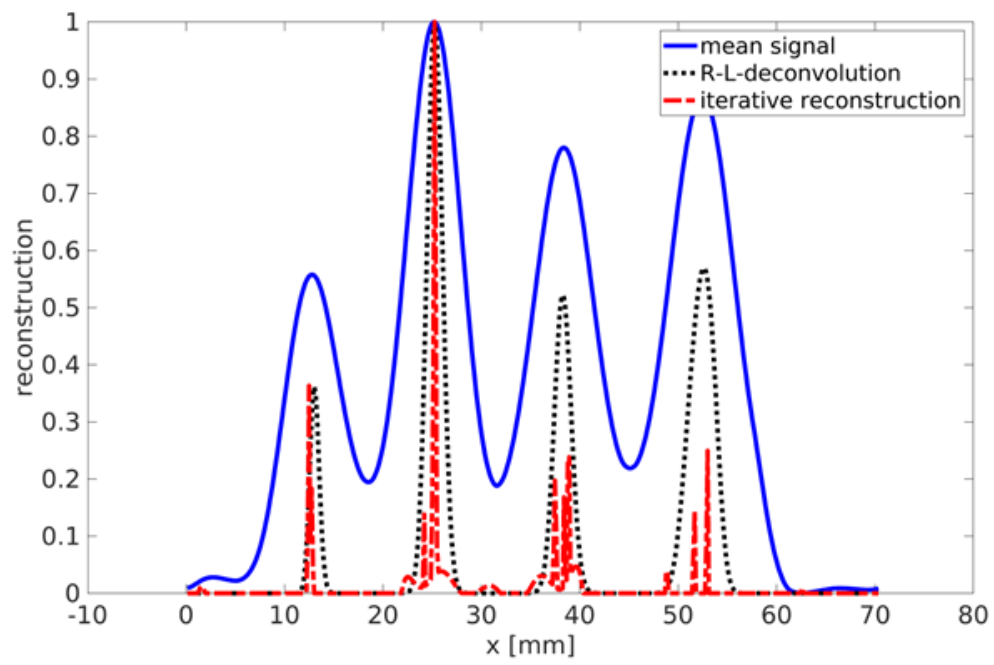

Fig. 7. Using $4.5 \mathrm{~mm}$ thick illumination lines instead of $1 \mathrm{~mm}$ in Fig. 5 reduces the spatial resolution significantly. Now the nearest line pair cannot be resolved. Only the line pairs having a distance of 0.9, 1.3, and $2 \mathrm{~mm}$ can be resolved.

For photothermal radiometry from one side in scattering media such as tissue by illumination with coherent laser light the speckles inside the tissue could be used as blind structured illumination. As the correlation time, that is the time before a new speckle pattern is established, is in living tissue only in the order of several milliseconds, laser pulses up to the $\mathrm{kHz}$ range could be used to generate different structured illumination. But this possibility presents additional challenges. For fully developed speckle in diffusive media, the speckle size is only about half the optical wavelength. Here, the variance of the acoustic signals for the different illumination patterns $I_{m}$ will be quite small [7]. It may be possible to overcome this through improvements in the signal-to-noise ratio of the radiometry measurements. The speckle size can also be increased by moving to a larger optical wavelength. In living tissue speckle decorrelation leads to additional challenges, as the speckle field changes with a characteristic time scale of milliseconds due to physiological motion; necessitating rapid data acquisition. Nevertheless, we believe that super-resolution thermographic imaging offers a promising new approach to achieve high-resolution imaging at depth in scattering media. 


\section{Acknowledgement}

This work was supported by the "K-Project for non-destructive testing and tomography plus" supported by COMET program of FFG and the federal government of Upper Austria and Styria and by the project "multimodal and insitu characterization of inhomogeneous materials" (MiCi) by the federal government of Upper Austria and the European Regional Development Fund (EFRE) in the framework of the EU-program IWB2020. We also acknowledge funding from the federal government of Upper Austria within the strategic-economic research program "Innovative Upper Austria 2020".

\section{REFERENCES}

[1] Burgholzer P., and Hendorfer G. Limits of spatial resolution for thermography and other non-destructive imaging methods based on diffusion waves. Int. J. Thermophys. Vol. 34, pp.1617-1632, 2013.

[2] Burgholzer P. Thermodynamic limits of spatial resolution in active thermography. J. Thermophys. Vol. $36, \mathrm{pp}$. 2328-2341, 2015.

[3] I.M. Vellekoop, A. Mosk, Focusing coherent light through opaque strongly scattering media, Optics letters vol. 32, pp. 2309-2311, 2007.

[4] Gustafsson M. G. Surpassing the lateral resolution limit by a factor of two using structured illumination microscopy. J. Microscopy vol. 198, pp.82-87, 2000.

[5] E. Mudry, K. Belkebir, J. Girard, J. Savatier, E. L. Moal, C. Nicoletti, M. Allain, and A. Sentenac, Structured illumination microscopy using unknown speckle patterns. Nat. Photon. 6, 312, 2012.

[6] J. Min, J.D. Jang, D.M. Keum, S.W. Ryu, C.H. Choi, K.H. Jeong, and J.C. Ye, "Fluorescent microscopy beyond diffraction limits using speckle illumination and joint support recovery," Sci. Rep 3, 2075, 2013.

[7] T. W. Murray, M. Haltmeier, T. Berer, E. Leiss-Holzinger, and P. Burgholzer, Optica vol. 4, 17, 2017.

[8] E. Hojman, T. Chaigne, O. Solomon, S. Gigan, E. Bossy, Y. C. Eldar, and O. Katz, Opt. Express vol. 25, 4875, 2017.

[9] Burgholzer P., Berer T., Gruber J., and Mayr G. Super-resolution thermographic imaging using blind structured illumination. Applied Physics Letters vol. 111, no. 3, pp. 031908-1-5, 2017.

[10] Burgholzer P., Thor M., Gruber J., and Mayr G. Three-dimensional thermographic imaging using a virtual wave concept. J. appl. Phys. Vol. 121, pp. 105102-1-11, 2017.

[11] A. Beck and M. Teboulle "A Fast Iterative Shrinkage-Thresholding Algorithm for Linear Inverse Problems," SIAM J. Imaging Sci. vol. 2, pp. 183-202, 2009.

[12] E. Thiel, M. Ziegler and T. Studemund. Int J Thermophys, "Localization of subsurface defects in uncoated aluminum with structured heating using high-power VCSEL laser arrays", submitted 2018.

[13] M. Ziegler, E. Thiel and T. Studemund. "Thermography using a 1D laser array - From planar to structured heating, Materials Testing", in print 2018. 